\title{
Polystyrene Microsphere Optical Properties by Kubelka-Munk and Diffusion Approximation with a Single Integrating Sphere System: A Comparative Study
}

\author{
Ali Shahin $\mathbb{D}^{1},{ }^{1}$ Wesam Bachir, ${ }^{1,2}$ and Moustafa Sayem El-Daher $\mathbb{D}^{3,4}$ \\ ${ }^{1}$ Biomedical Photonics Laboratory, Higher Institute for Laser Research and Applications, Damascus University, \\ Damascus, Syria \\ ${ }^{2}$ Faculty of Informatics Engineering, Al-Sham Private University, Damascus, Syria \\ ${ }^{3}$ Higher Institute for Laser Research and Applications, Damascus University, Damascus, Syria \\ ${ }^{4}$ Faculty of Informatics and Communications, Arab International University, Daraa, Syria \\ Correspondence should be addressed to Ali Shahin; ali.shahin88@yahoo.com
}

Received 25 September 2019; Revised 26 October 2019; Accepted 14 November 2019; Published 1 December 2019

Academic Editor: Daniel Cozzolino

Copyright (c) 2019 Ali Shahin et al. This is an open access article distributed under the Creative Commons Attribution License, which permits unrestricted use, distribution, and reproduction in any medium, provided the original work is properly cited.

\begin{abstract}
The optical properties of $1 \mu \mathrm{m}$ polystyrene in the wavelength range of $500-750 \mathrm{~nm}$ were estimated by using a white light spectrophotometric transmittance spectroscopy and a single integrating sphere system. To retrieve the optical characteristics, two analytical methods, namely, diffusion approximation and Kubelka-Munk were used, and then their results were compared with Mie theory calculations. The correspondence of the Kubelka-Munk scattering coefficient with Mie was obvious, and relative errors varied between $6.73 \%$ and $2.66 \%$ whereas errors varied between $6.87 \%$ and $3.62 \%$ for diffusion theory. Both analytical methods demonstrated the absorption property of polystyrene over the abovementioned wavelength range. Although absorption coefficient turned out to be much lower than scattering, constructing a realistic optical phantom requires taking into account absorption property of polystyrene. Complex refractive index of polystyrene based on these two methods was determined. Inverse Mie algorithm with scattering coefficient was also used to retrieve the real part of refractive index and absorption coefficient for calculating the imaginary part of refractive index. The relative errors of the real part did not exceed $2.6 \%$, and the imaginary part was in consistence with the prior works. Finally, the presented results confirm the validity of diffusion theory with a single integrating sphere system.
\end{abstract}

\section{Introduction}

Optical characterization techniques require both an experimental setup to measure the radiometric characteristics and a light propagation method to extract the macroscopic optical properties. Generally, these techniques are categorized as direct and indirect methods $[1,2]$. The direct method can be performed only ex vivo, and the samples used in this technique are thin enough so that the multiple scattering events can be negligible, but the advantage of this method depends on simple exponential equations to extract the optical properties [2]. In contrast, indirect methods can be implemented ex vivo and in vivo with thick samples [2-5]. Unfortunately, the estimation of the optical properties via these methods is based on more complicated mathematical principles and analytical and numerical solutions of the radiative transfer equation (RTE) [1-4]. Kubelka-Munk may be considered as the simplest analytical method that has been used for optical characterization, but the drawback of this method is limited to the case of highly scattering medium, and the sample's thickness should exceed the transport length. Also, this model does not take into account the jump in the refractive index between the sample and surrounding medium $[6,7]$. Recently, this approach has been modified to overcome the refractive index mismatch between the sample and holder, and the thickness does not exceed the transport length [7]. The present method has been investigated with a single integrating sphere system 
over visible and $\mathrm{N}$-IR wavelength range on biological tissues and optical phantoms $[7,8]$. On the other hand, diffusion approximation has been considered as an approximate solution of the radiative transfer equation for semi-infinite and high diffusive medium for collimated incident light $[9,10]$. That has been used as a quantitative method to retrieve the optical properties of several media using diffuse reflectance spectra which supplies a correlation of measurements with some constraints, i.e., a highly diffusive medium with a large distance between the semi-infinite sample and source. Accordingly, diffusion approximation has been used in optical characterization that has been in agreement to theoretical models such as Mie theory and the Monte Carlo method $[11,12]$. Also, this approach has been used with an integrating sphere method in vivo with a large medium to simulate the boundary conditions of this model $[13,14]$.

On the other hand, polystyrene microsphere has been widely used as a tissue-simulating phantom component to mimic scattering property of a certain tissue. The ability to estimate its optical characteristics accurately via Mie theory calculation that provides a level of validation does not exist in any other optical phantom component. However, most prior research groups deal with this material as a pure scattering without taking into account absorption property and retrieved scattering coefficient by using Mie theory $[15,16]$. Thus, precise optical characterization of these materials has an enormous effect on constructing ideal phantoms [15].

The aim of this research is to investigate the validity of diffusion approximation with a single integrating sphere system compared with analytical and theoretical approaches. Thus, albedo, scattering coefficient, extinction coefficient, and complex refractive index of a $1 \mu \mathrm{m}$ polystyrene microsphere (07310-15, Polybead, Polysciences, USA) were determined by using diffusion approximation and Kubelka-Munk based on radiometric measurements using a single integrating sphere system and collimated transmission spectroscopy. Then, Mie theory calculation was implemented to compare its scattering coefficient with the two models' results which was considered as a standard method for a spherical particle like polystyrene. Finally, complex refractive index of a $1 \mu \mathrm{m}$ polystyrene was determined in the range of wavelength $500-750 \mathrm{~nm}$ based on these two models' scattering coefficient and inverse Mie theory calculation.

\section{Materials and Methods}

2.1. Diffusion Approximation Method. Diffusion equation is a differential equation for fluence rate which can be derived from a radiative transfer equation depending on some assumptions as a highly homogenous scattering property for a semi-infinite medium. For a collimated beam incident normally on a surface and taking into account the refractive index mismatch, the diffuse reflectance $R_{\mathrm{d}}$ from a semiinfinite medium can be expressed as a function of a reduced albedo $a^{\prime}$ and an internal diffuse reflectance $r$, as follows $[10,13]$ :

$$
R_{\mathrm{d}}=\frac{a^{\prime}}{1+2 \cdot K\left(1-a^{\prime}\right)+(1+(2 \cdot K / 3)) \cdot \sqrt{3 \cdot\left(1-a^{\prime}\right)}}
$$

where $a^{\prime}=\mu_{s}^{\prime} /\left(\mu_{s}^{\prime}+\mu_{a}\right)$ is the reduced albedo and $k=(1+r) /$ $(1-r)$ is a factor related to internal diffuse reflectance $r$ which is given by $[10,13]$

$$
\begin{aligned}
r & =-1.44 n_{\text {rel }}^{-2}+0.71 n_{\text {rel }}^{-1}+0.668+0.0636 n_{\text {rel }}, \\
n_{\text {rel }} & =\frac{n_{\text {sample }}}{n_{\text {surrounding meduim }}},
\end{aligned}
$$

where $n_{\text {rel }}$ is the relative refractive index, $n_{\text {sample }}$ is the polystyrene refractive index, and $n_{\text {surroundingmeduim }}$ is the quartz cuvette refractive index $[10,13]$.

2.2. Kubelka-Munk Model. Kubelka-Munk theory is an analytical solution of a radiative transfer equation (RTE) based on some presumptions. The simplicity of the Kubelka-Munk method has been the main reason for its widespread uses $[6,7]$. This theory introduced special coefficients: $K$ is a Kubelka-Munk absorption coefficient and $S$ is a Kubelka-Munk scattering coefficient, which are related to macroscopic optical coefficients as follows $[1,6]$ :

$$
\begin{aligned}
\frac{K}{S} & =\frac{\left(1-R_{\infty}\right)^{2}}{2 \cdot R_{\infty}}, \\
K & =2 \cdot \mu_{a}, \\
S & =\frac{3}{4} \cdot \mu_{s}^{\prime}-\frac{1}{4} \cdot \mu_{a} .
\end{aligned}
$$

This model has been studied and tested extensively by several research groups on chemical substances as well as biological tissues. Recently, a modified approach has been developed and implemented on tissues and optical phantom components with a single integrating sphere system [7]. The modified method takes into account the mismatch in the refractive index between sample and holder by using a Fresnel transmission coefficient expressed as follows [7]:

$$
T_{\mathrm{f}}=\frac{4 \cdot n}{(n+1)^{2}},
$$

where $n$ is the refractive index of the sample. Scattering and absorption coefficients can be retrieved by measuring diffuse transmission and reflection spectra which are given by these correlations [7]:

$$
\begin{aligned}
& \mu_{a}=\frac{1}{d} \log \frac{T_{\mathrm{f}}^{2}}{T_{\mathrm{d}}}-\frac{2 \cdot R_{\mathrm{d}} \cdot T_{\mathrm{f}}^{2} \cdot \log \left(T_{\mathrm{f}}^{2} / T_{\mathrm{d}}\right)}{d \cdot\left(T_{\mathrm{f}}^{4}-T_{\mathrm{d}}^{2}\right)}, \\
& \mu_{s}=\frac{1}{d} \log \frac{T_{\mathrm{d}}}{T_{\mathrm{c}}}+\frac{2 \cdot R_{\mathrm{d}} \cdot T_{\mathrm{f}}^{2} \cdot \log \left(T_{\mathrm{f}}^{2} / T_{\mathrm{d}}\right)}{d \cdot\left(T_{\mathrm{f}}^{4}-T_{\mathrm{d}}^{2}\right)},
\end{aligned}
$$

where $T_{\mathrm{d}}$ is the diffuse transmittance, $R_{\mathrm{d}}$ is the diffuse reflectance, $T_{c}$ is the collimated transmittance, and $d$ is the sample thickness. Since a small concentration of polystyrene 
was used, the refractive index can be considered as a refractive index of the solvent.

2.3. Mie Theory Calculation. Mie theory is an analytical solution of Maxwell's equations for the scattering of electromagnetic radiation by a single spherical particle. It provides an exact solution for the scattering and the anisotropy coefficients of perfect spheres [17]. Matzler computer program was used to calculate Mie scattering parameters, i.e., scattering efficiency $Q\left(r_{i}, \lambda\right)$ and anisotropy coefficient $g\left(r_{i}, \lambda\right)$ that were used to extract the scattering coefficient $\mu_{s}(\lambda)$ and anisotropy factor $g(\lambda)[18,19]$. This method requires particle diameter, fraction, and wavelength of radiation in the vacuum besides refractive index of the particle and refractive index of the solvent $[18,19]$. A pure polystyrene sample was an aqueous suspension (07310-15, Polybead, Polysciences, USA), and the fraction of $1 \mu \mathrm{m}$ polystyrene particles was $2.5 \%(\mathrm{w} / \mathrm{v})$. In addition, refractive indices of polystyrene as a scattering and water as a host material were found from previous work [20].

\subsection{Spectrophotometric Transmission Spectroscopy. A} broadband, fiber-based spectrophotometric transmission setup was arranged. That consists of a broadband halogentungsten light source (HL-2000-HP-FHSA, Ocean Optics Inc., FL), a fiber-coupled cuvette holder with two collimating lenses (CUV-ATT-DA, Avantes Inc., Netherlands), and a USB portable spectrometer (USB4000 FL, Ocean Optics Inc.). The extinction coefficient of polystyrene samples can be measured within a broad wavelength range from 500$750 \mathrm{~nm}$. The sample was illuminated with a collimated white light beam. Thus, the transmission intensity may be approximated by the Beer-Lambert Law $[8,9]$ :

$$
\begin{aligned}
& I_{z}(\lambda)=I_{0}(\lambda) \cdot e^{-\mu_{t}(\lambda) \cdot z \cdot c}, \\
& \frac{I_{z}(\lambda)}{I_{0}(\lambda)}=T_{c},
\end{aligned}
$$

where $\mu_{t}(\lambda)$ is the extinction coefficient, $I_{z}(\lambda)$ is the intensity of light passed through the studied sample, $I_{0}(\lambda)$ is the reference intensity, $z$ is the optical path length (thickness of cuvette), $c$ is the concentration, and $T_{c}$ is the collimated transmittance $[8,9]$.

2.5. Integrating Sphere System. A broadband single integrating sphere (819C-IS-5.3, Newport, USA) system was used. A schematic drawing of diffuse reflection measurement using a single integrating sphere system is shown in Figure 1(a). Diffuse transmittance measurement via an integrating sphere can be seen from Figure 1(b). Light from a tungsten-halogen lamp (HL-2000-HP-FHSA, Ocean Optics Inc., FL) was delivered to the sample of interest through a convex lens with a $10 \mathrm{~cm}$ focal length and a $1.5 \mathrm{~mm}$ pinhole, and the diffuse light emanated from the sample is guided through an optical fiber to the spectrometer (USB4000 FL, Ocean Optics, USA).
The fiber optic spectrometer is connected to a computer for data acquisition and further analysis. The spectrum was acquired in the range between 500 to $750 \mathrm{~nm}$ with the help of the SpectraSuite software (version 2011, Ocean Optics, USA). Dark current and background measurements were taken at the same setting before each recording [21, 22].

\section{Results}

Based on collimated transmission spectra extracted by spectrophotometric transmission spectroscopy and the Beer-Lamber law, equation (7), the extinction coefficient of $1 \mu \mathrm{m}$ polystyrene microsphere can be calculated over the wavelength range of $500-750 \mathrm{~nm}$, as shown in Figure 2. Obviously, the extinction coefficient was found to be inversely proportional to the increased wavelength over the studied range of wavelength, Figure 2. Using the curve fitting procedure in Matlab 2014, the correlation of PS extinction coefficient and wavelength could be found and given by

$$
\mu_{t}=1.7 * 10^{6} \cdot \lambda^{-1.498}
$$

3.1. Mie Theory. Mie theory is considered as an exact solution of the Maxwell equations for the scattering of electromagnetic radiation by spherical particles such as polystyrene microsphere (07310-15, Polybead, Polysciences, USA). Refractive index of PS was found from the prior work, and for water (host material), it was about 1.33 [20]. Depending on a polystyrene datasheet from a manufacturer and Matzler computer program, scattering coefficient and anisotropy factor could be predicted $[18,19]$. Mie scattering coefficient was found to be inversely proportional to increased wavelength, as shown in Figure 3(a). Using anisotropy factor $g$ and Mie scattering coefficient, reduced scattering coefficient could be found. These relations were power equations estimated via the curve fitting procedure in Matlab and given by

$$
\begin{aligned}
& \mu_{s_{-} \text {Mie }}=1.7 * 10^{6} \cdot \lambda^{-1.513}, \\
& \mu_{s_{-} \text {Mie }}^{\prime}=3689 \cdot \lambda^{-0.9462} .
\end{aligned}
$$

3.2. Kubelka-Munk. The modified Kubelka-Munk approach estimates scattering coefficient based on collimated transmittance $T_{\mathrm{c}}$, diffuse reflectance $R_{\mathrm{d}}$, and diffuse transmittance $T_{\mathrm{d}}$, as shown in equation (6). Using spectrophotometric transmission spectroscopy and a single integrating sphere system, PS radiometric characteristics could be measured. Then, Kubelka-Munk scattering coefficient could be evaluated, as shown in Figure 4(a). Accordingly, K-M scattering coefficient was plotted with wavelength, and that was found to be inversely proportional to increased wavelength. The correlations of $\mathrm{K}-\mathrm{M}$ scattering and reduced scattering coefficients and wavelength were found using the curve fitting procedure which were compatible with Mie relations, as follows: 


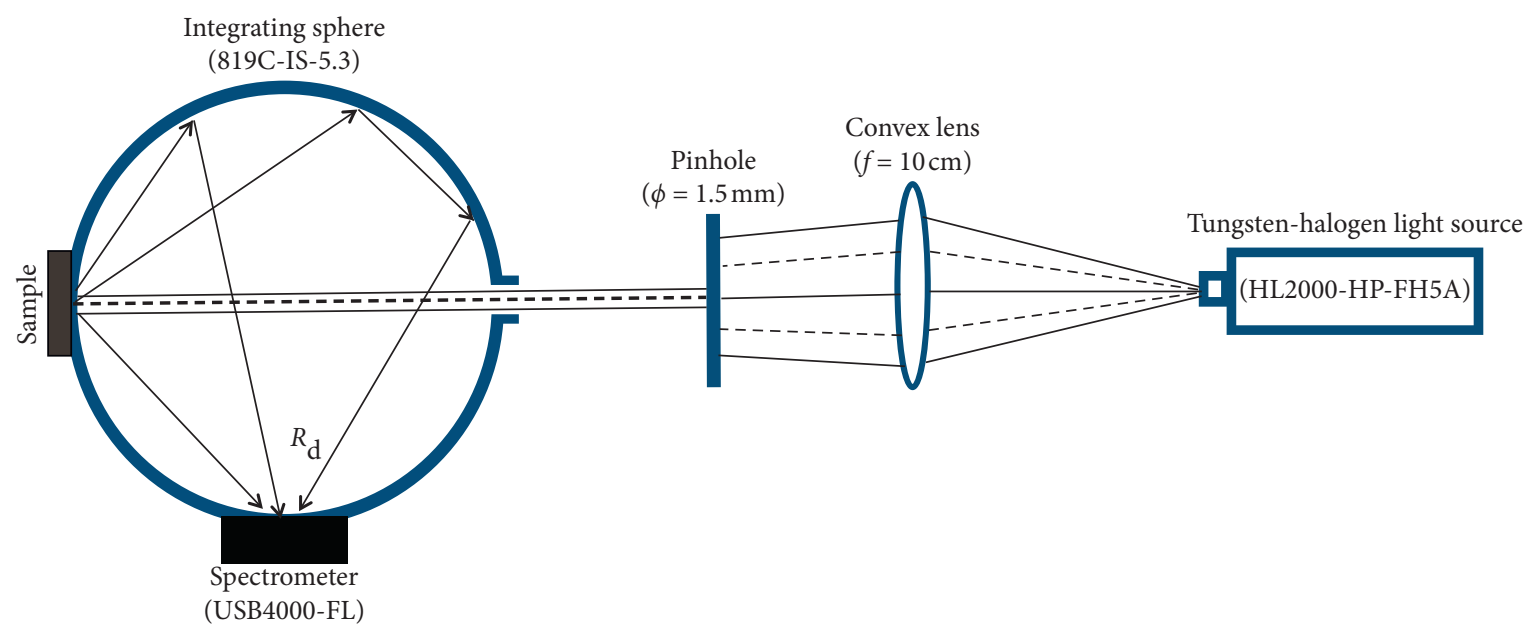

(a)

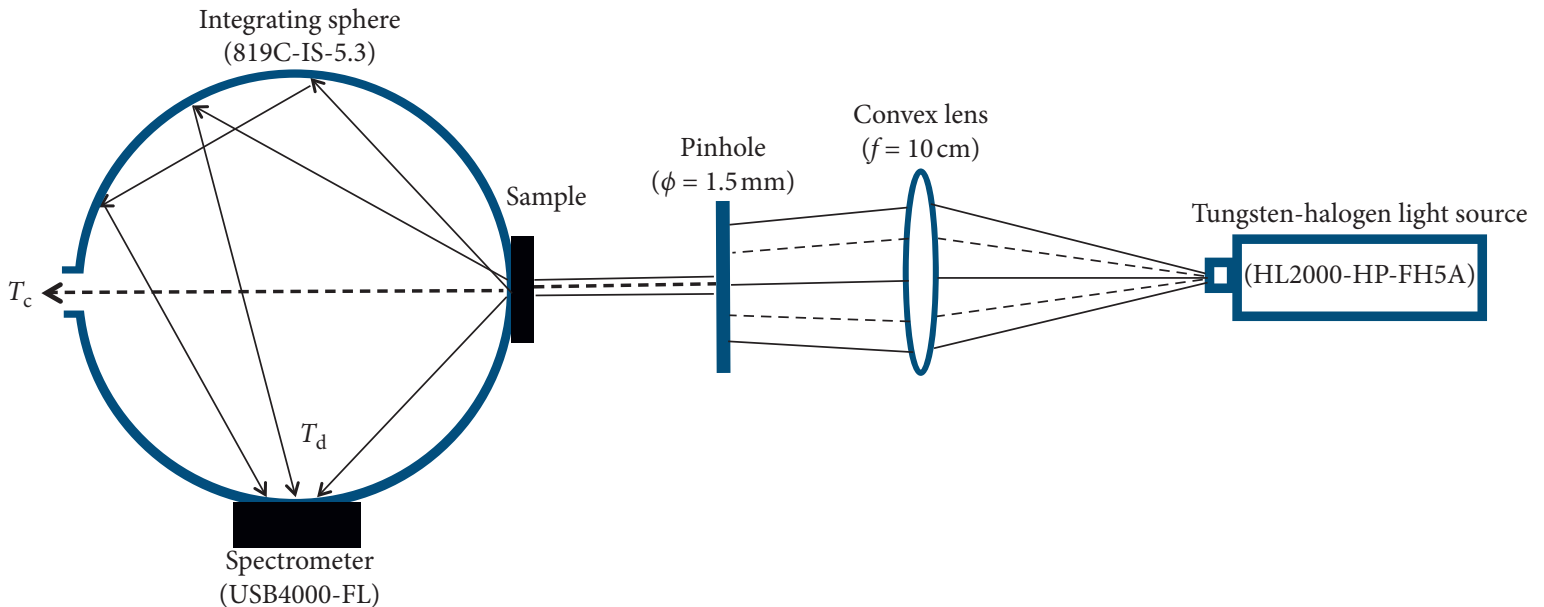

(b)

Figure 1: (a) Schematic drawing of the diffuse reflection measurement setup and (b) schematic drawing of the diffuse transmission measurement setup.

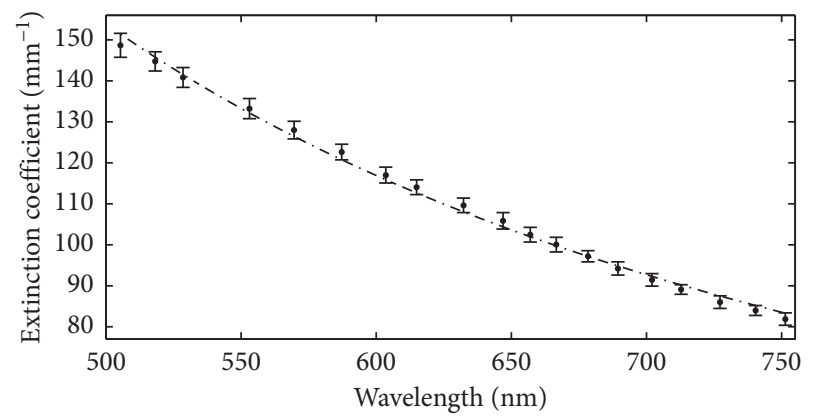

FIGURE 2: Correlation of the extinction coefficient calculated by collimated transmission spectroscopy and wavelength.

$$
\begin{aligned}
& \mu_{s_{-} \mathrm{KM}}=1.7 * 10^{6} \cdot \lambda^{-1.507}, \\
& \mu_{s_{-} \mathrm{KM}}^{\prime}=3689 \cdot \lambda^{-0.9405} .
\end{aligned}
$$

Based on extinction coefficient of PS and K-M scattering results, $\mathrm{K}-\mathrm{M}$ albedo (blue dotted line) might be calculated, Figure 5(b). Then, using inverse Mie calculation and
Kubelka-Munk scattering coefficient, the real part of refractive index could be estimated over the present wavelength range, Figure 5(c). Then, the wavelength dependence of the real part of refractive index for Kubelka-Munk (blue dotted line) was fitted to the Cauchy relation:

$$
n_{r}(\lambda)=1.61-\frac{0.02742}{\lambda^{2}}+\frac{0.008074}{\lambda^{4}},
$$

where $\lambda$ is estimated in micrometer. Then, $\mathrm{K}-\mathrm{M}$ absorption coefficient might be utilized to predict an imaginary part of refractive index using the following equation, Figure 5(d):

$$
n_{i}=\frac{\mu_{a} \cdot \lambda}{4 \pi}
$$

3.3. Diffusion Theory. Diffusion approximation introduces diffuse reflectance $R_{\mathrm{d}}$ as a function of reduced albedo $a^{\prime}$ and relative refractive index $n_{\text {rel }}$, equation (1). Based on diffuse reflectance measured via a single integrating sphere system and relative refractive index, reduced albedo could be 


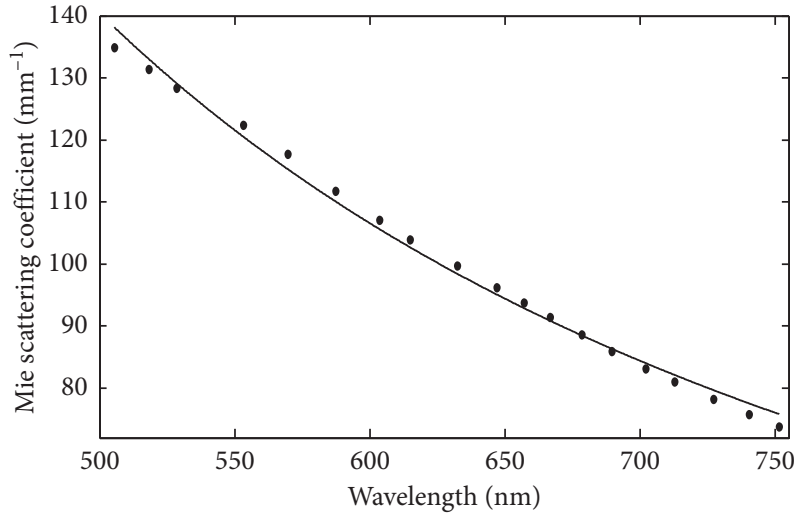

(a)

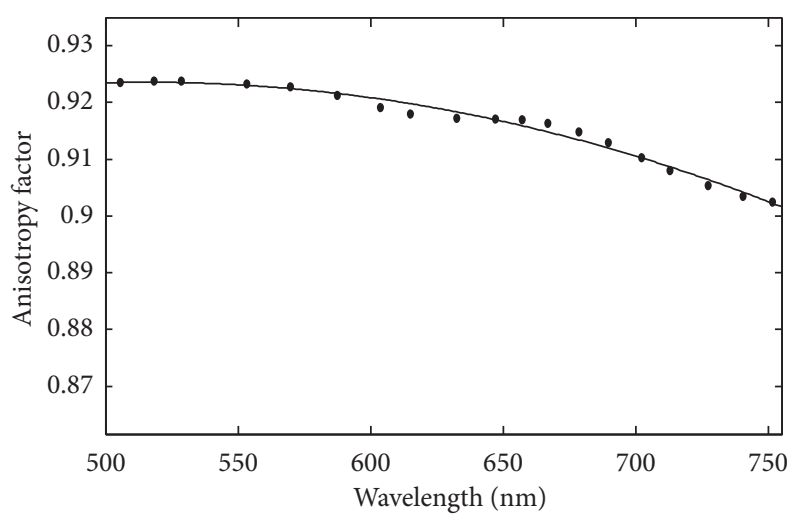

(b)

Figure 3: (a) Correlation of Mie scattering coefficient and wavelength and (b) anisotropy factor calculated using Mie theory with wavelength.

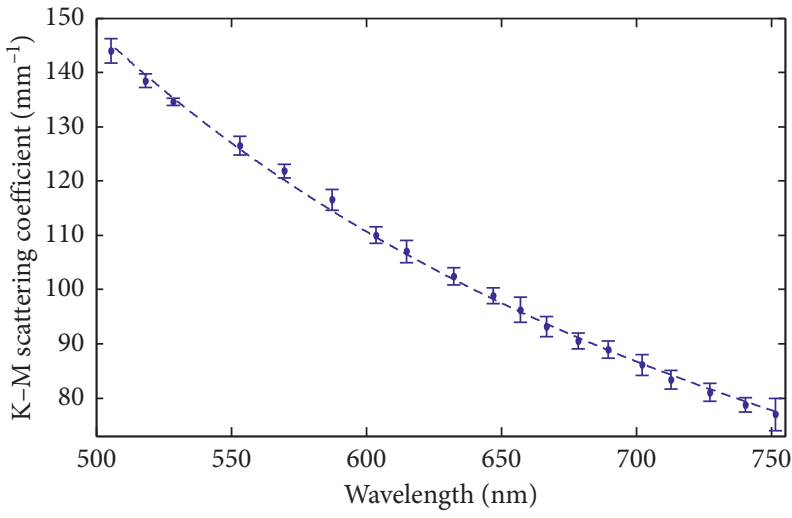

(a)

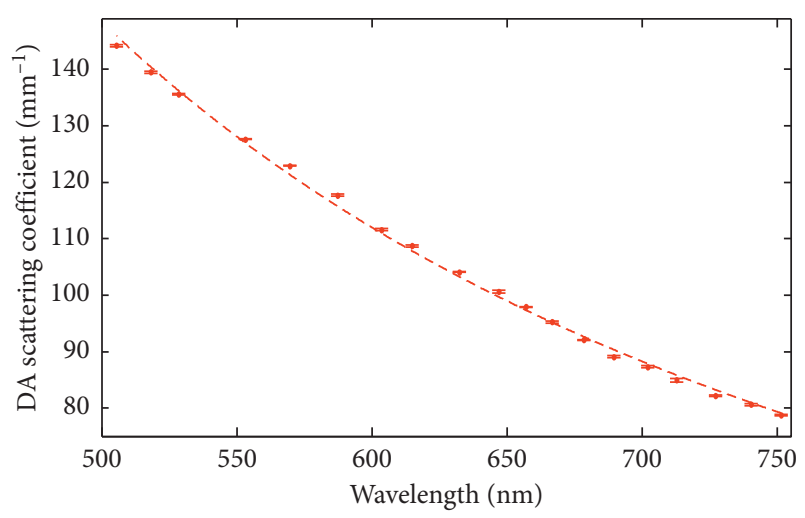

(b)

Figure 4: (a) Correlation of Kubelka-Munk scattering coefficient and wavelength and (b) relation between diffusion approximation scattering coefficient with wavelength.

evaluated. Using reduced albedo, extinction coefficient estimated by collimated transmittance spectroscopy and anisotropy factor predicted by Mie theory calculation, DA scattering coefficient (red dashed line) might be calculated, Figure 4(b). Also, DA scattering coefficient was found to be inversely proportional to increased wavelength, Figure 4(b). That was obvious using the curve-fitting procedure in Matlab 2014 which was a power correlation given by

$$
\begin{aligned}
& \mu_{s_{-} \mathrm{DA}}=1.7 * 10^{6} \cdot \lambda^{-1.505}, \\
& \mu_{s_{-} \mathrm{DA}}^{\prime}=3689 \cdot \lambda^{-0.9386} .
\end{aligned}
$$

Depending on PS extinction and DA scattering coefficients, DA albedo could be evaluated, Figure 5(b). It can be seen from Figure 5(b) that DA albedo (red dashed line) was found to be unsystematically changed with wavelength. Then, the real part of refractive index might be calculated based on DA scattering coefficient and inverse Mie calculation, Figure 5(c). Correlation of the DA real part of refractive index (red dashed line) and wavelength was fitted to the Cauchy equation as follows:

$$
n_{r}(\lambda)=1.611-\frac{0.02574}{\lambda^{2}}+\frac{0.007688}{\lambda^{4}} .
$$

Using equation (13) and DA absorption coefficient, the imaginary part of refractive index could be estimated. The relationship between DA imaginary part of refractive index and wavelength could be seen in Figure 5(d).

\section{Discussion}

Spectrophotometric transmission spectroscopy was used to measure the collimated transmittance of a $1 \mu \mathrm{m}$ polystyrene utilized to estimate the extinction coefficient, Figure 2. From Figure 2 and equation (9), the power correlation of the extinction coefficient and wavelength over a studied range were obvious. Based on diffuse transmittance and reflectance that were measured by a single integrating sphere system and collimated transmittance, the scattering coefficient of $1 \mu \mathrm{m}$ polystyrene sphere could be predicted using Kubelka-Munk and diffusion approximation methods, Figures 4(a) and 4(b), respectively. As can be seen from equations (11) and (14), 

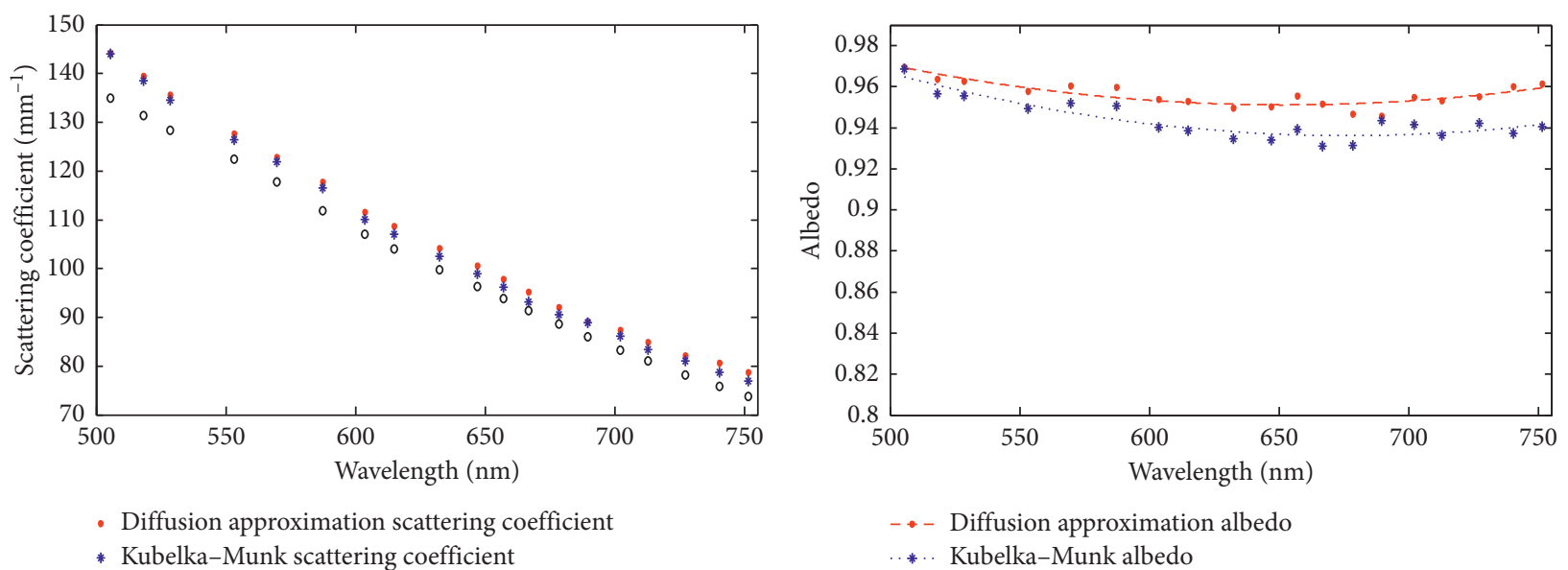

- Diffusion approximation scattering coefficient

* Kubelka-Munk scattering coefficient

- Mie theory scattering coefficient

(a)
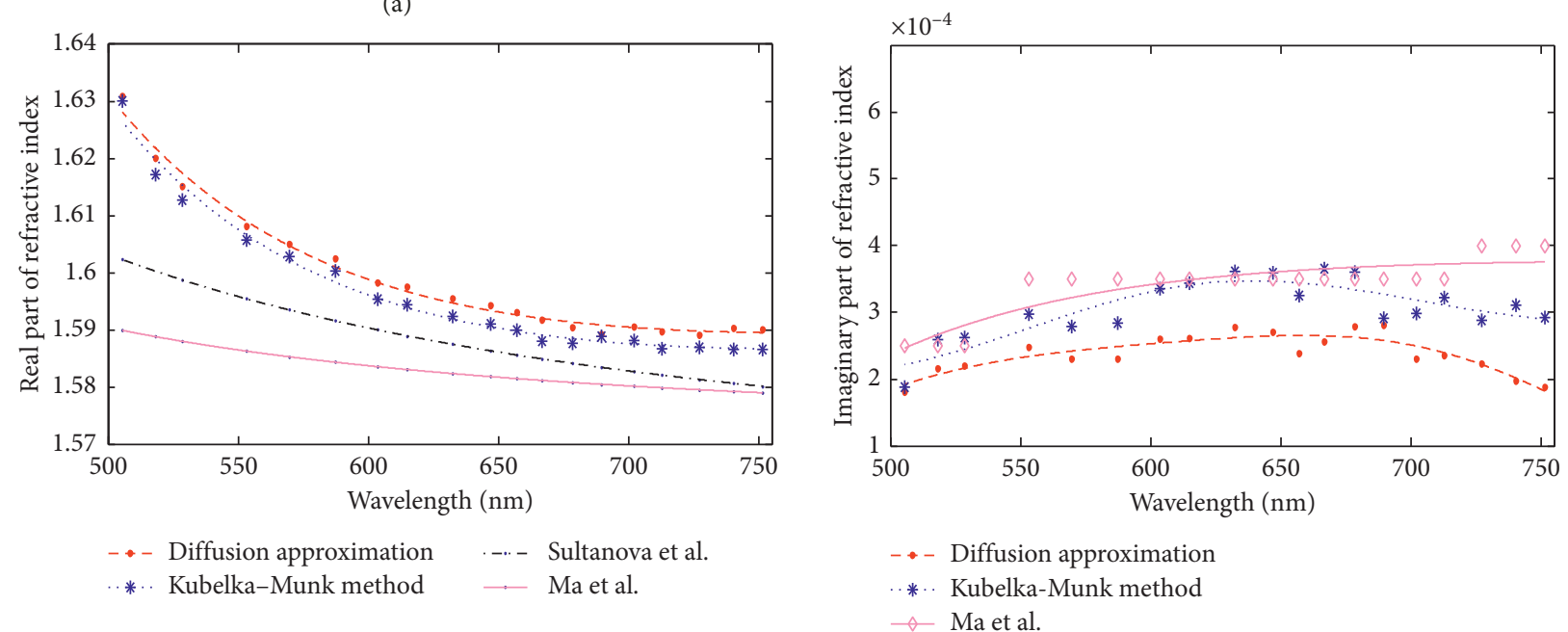

(c)

(d)

Figure 5: (a) Comparison between KM, DA, and Mie scattering over the wavelength. (b) Correlation of DA (red) and KM (blue) albedo with wavelength. (c) Correlation of the real part of refractive index estimated by diffusion approximation (red) and Kubelka-Munk (blue) with wavelength. (d) Relation between the imaginary part of refractive index calculated by diffusion approximation (red) and Kubelka-Munk (blue) with wavelength.

Kubelka-Munk and diffusion approximation scattering were found to be inversely proportional to the wavelength that were in consistence with Mie calculation, and their power factor was about -1.505 for diffusion theory, -1.507 for the K-M model, and -1.513 for Mie calculation. This can be explained by the small incident wavelength in comparison with polystyrene diameter [17]. In addition, K-M scattering was in agreement with Mie calculation and the relative errors varied between $6.73 \%$ to $2.66 \%$, but scattering coefficient estimated by diffusion approximation turned out to be higher, and low absorption property could be noticed that was obvious from albedo values, Figure 5(b). DA relative errors varied between $6.87 \%$ and $3.62 \%$. The small difference between two methods' results could be explained by some reasons. The first one is that the thickness of a sample used in the experiment is about $1 \mathrm{~cm}$ which exceeds the transport length of incident light, and using a convex lens makes a diameter of beam to not exceed the dimension of the sample; all these points make the tested sample simulate approximately the semi-infinite medium which has been in agreement with using a diffusion approximation and not Kubelka-Munk [7, 10,13]. The second one is that a modified Kubelka-Munk method has been introduced in a case of a single scattering event, and the use of a polystyrene microsphere sample and a $1 \mathrm{~cm}$ sample thickness without adding any absorption materials makes the restriction unfulfilled, and multiple scattering event is dominant in the medium that is in agreement with diffusion approximation conditions $[7,10,13]$. Finally, the anisotropy factor has been estimated by Mie calculation that was about 0.9 as shown in Figure 3(b), and using this sample with a high anisotropy factor was in correspondance with a condition of deriving a diffusion approximation equation, equation (1), [10]. The findings presented in the previous section explain the applicability of diffusion approximation with an integrating sphere system and polystyrene sample. These methods, 
which were used in this paper, make it possible to reconstruct the absorption coefficient of a given sample, Figure 5(b). It should be noted that $\mathrm{K}-\mathrm{M}$ absorption property was higher compared to diffusion results. By comparison with absorption coefficient of water, $1 \mu \mathrm{m}$ polystyrene absorption property could not be neglected $[23,24]$. It can be seen from Figure 5(b) that the variation of albedo with wavelength was about 0.95 for the $\mathrm{K}-\mathrm{M}$ method and higher for diffusion. Thus, polystyrene microsphere can be considered as a turbid medium with highly scattering events in comparison with absorption property. In addition, the ratio between scattering and absorption over the present wavelength range varied from 30.69 at $505 \mathrm{~nm}$ to 13.52 at $665 \mathrm{~nm}$ based on Kubelka-Munk and from 32.03 at $505 \mathrm{~nm}$ to 19.71 at $665 \mathrm{~nm}$ for the diffusion method. Therefore, the absorption property of $1 \mu \mathrm{m}$ polystyrene microsphere could not be neglected when the ratio of scattering and absorption coefficient of a certain tissue is comparable with the later values. Furthermore, when the ratio of scattering and absorption coefficients equals the later ratios, the polystyrene can mimic optical properties of the given tissue only without adding any absorbing material.

Based on scattering and absorption coefficients of these methods and inverse Mie algorithm, the complex refractive index of $1 \mu \mathrm{m}$ polystyrene microsphere could be evaluated, Figures 5(c) and 5(d). As can be seen from Figure 5(c), the correlation of the real part of refractive index for these two methods with wavelength has similar trends that could be seen in equations (12) and (15). Also, K-M real refractive index seems to be lower whereas its imaginary part is higher. That difference could be explained by the mismatch between two models' optical properties. Based on a deviation angle method for refractive index measurement of polystyrene, the systematic errors of real refractive index calculations using $\mathrm{K}-\mathrm{M}$ and diffusion theory were estimated to be compared with prior research studies [20, 25, 26]. For Kubelka-Munk values, the relative reconstructed error varies between $1.8 \%$ to $0.2 \%$ and from $1.9 \%$ to $0.38 \%$ for diffusion theory results $[20,25,26]$. Inverse Mie calculation based on Monte Carlo algorithm was used to reconstruct the real refractive index of polystyrene, and the relative error of the Kubelka-Munk method varies between $2.52 \%$ and $0.43 \%$ and between $2.57 \%$ and $0.55 \%$ for diffusion theory results [24]. Furthermore, two approaches' calculations show a relatively high error in the lower wavelength, and the small errors could be seen at $600 \mathrm{~nm}[20,24-26]$. For the imaginary part of polystyrene refractive index, a similar behavior of $n_{i}$ variation with wavelength could be seen as shown in Figure 5(d), but diffusion theory $n_{i}$ turned out to be lower due to the small absorption property than $\mathrm{K}-\mathrm{M} n_{i}$ values. However, the relative error for the imaginary part of refractive index varies between 3\% and 30\% for Kubelka-Munk and between 10\% and $35 \%$ for diffusion theory [24].

\section{Conclusions}

In summary, the results given in this paper were an attempt to investigate the optical properties of $1 \mu \mathrm{m}$ polystyrene microsphere as a scattering material in solid and liquid optical phantoms. For this purpose, Kubelka-Munk and diffusion approximation methods were used to calculate the optical properties of polystyrene. Then, results confirm the accuracy of the results and applicability of diffusion theory with the present experimental limitations.

On the one hand, the applicability of using diffusion theory has been constrained by many factors. This theory could be used with a semi-infinite diffusive medium and collimated beam simulated by a thick scattering sample. Moreover, anisotropy factor of the sample should not exceed 0.95 whereas Kubelka-Munk could be applied for any given medium.

On the other hand, it can be seen that polystyrene showed an absorption effect that was small in comparison with the scattering effect and varied over the spectral range. The decisive factor for neglecting polystyrene absorption depends on the ratio of scattering and absorption of a given medium.

\section{Data Availability}

The data used to support the findings of this study are available from the corresponding author upon request.

\section{Conflicts of Interest}

The authors declare that they have no conflicts of interest.

\section{References}

[1] B. C. Wilson, M. S. Patterson, and S. T. Flock, "Indirect versus direct techniques for the measurement of the optical properties of tissues," Photochemistry and Photobiology, vol. 46, no. 5, pp. 601-608, 1987.

[2] A. N. Bashkatov, E. A. Genina, and V. V. Tuchin, "Optical properties of skin, subcutaneous, and muscle tissues: a review," Journal of Innovative Optical Health Sciences, vol. 4, no. 1, pp. 9-38, 2011.

[3] J. L. Sandell and T. C. Zhu, "A review of in-vivo optical properties of human tissues and its impact on PDT," Journal of Biophotonics, vol. 4, no. 11-12, pp. 773-787, 2011.

[4] A. N. Yaroslavsky, P. C. Schulze, I. V. Yaroslavsky, R. Schober, F. Ulrich, and H.-J. Schwarzmaier, "Optical properties of selected native and coagulated human brain tissues in vitro in the visible and near infrared spectral range," Physics in Medicine and Biology, vol. 47, no. 12, pp. 2059-2073, 2002.

[5] H. Soleimanzad, H. Gurden, and F. Pain, "Optical properties of mice skull bone in the 455-to 705-nm range," Journal of Biomedical Optics, vol. 22, no. 1, Article ID 010503, 2017.

[6] S. N. Thennadil, "Relationship between the Kubelka-Munk scattering and radiative transfer coefficients," Journal of the Optical Society of America A, vol. 25, no. 7, pp. 1480-1485, 2008.

[7] A. D. Krainov, A. M. Mokeeva, E. A. Sergeeva, P. D. Agrba, and M. Y. Kirillin, "Optical properties of mouse biotissues and their optical phantoms," Optics and Spectroscopy, vol. 115, no. 2, pp. 227-234, 2013.

[8] A. Shahin, M. Sayem El-Daher, and W. Bachir, "Determination of the optical properties of Intralipid $20 \%$ over a broadband spectrum," Photonics Letters of Poland, vol. 10, no. 4, pp. 124-126, 2018. 
[9] A. Shahin and W. Bachir, "Broadband spectroscopy for characterization of tissue-like phantom optical properties," Polish Journal of Medical Physics and Engineering, vol. 23, no. 4, pp. 121-126, 2017.

[10] S. T. Flock, M. S. Patterson, B. C. Wilson, and D. R. Wyman, "Monte Carlo modeling of light propagation in highly scattering tissues. I: model predictions and comparison with diffusion theory," IEEE Transactions on Biomedical Engineering, vol. 36, no. 12, pp. 1162-1168, 1989.

[11] A. T. Lovell, J. C. Hebden, J. C. Goldstone, and M. Cope, "Determination of the transport scattering coefficient of red blood cells," in Proceedings of the SPIE, vol. 3597, San Jose, CA, USA, July 1999.

[12] R. Michels, F. Foschum, and A. Kienle, "Optical properties of fat emulsions," Optics Express, vol. 16, no. 8, pp. 5907-5925, 2008.

[13] A. J. Welch and M. J. C. van Gemert, Optical-Thermal Response of Laser-Irradiated Tissue, Springer, New York, NY, USA, 2011.

[14] P. R. Bargo, S. A. Prahl, T. T. Goodell et al., "In vivo determination of optical properties of normal and tumor tissue with white light reflectance and an empirical light transport model during endoscopy," Journal of Biomedical Optics, vol. 10, no. 3, Article ID 034018, 2005.

[15] B. W. Pouge and M. S. Patterson, "Review of tissue simulating phantoms for optical spectroscopy, imaging and dosimetry," Journal of Biomedical Optics, vol. 11, no. 4, Article ID 041102, 2006.

[16] Y. Liu, Y. L. Kim, and V. Backman, "Development of a bioengineered tissue model and its application in the investigation of the depth selectivity of polarization gating," Applied Optics, vol. 44, no. 12, pp. 2288-2299, 2005.

[17] H. C. van de Hulst, Light Scattering by Small Particles, Dover Publication, New York, NY, USA, 1981.

[18] C. Matzler, Matlab Functions for Mie Scattering and Absorption, Bern University, Bern, Germany, 2002.

[19] C. Matzler, Matlab Functions for Mie Scattering and Absorption, Version 2, Bern University, Bern, Germany, 2002.

[20] N. Sultanova, S. Kasarova, and I. Nikolov, "Dispersion properties of optical polymers," Acta Physica Polonica A, vol. 116, no. 4, pp. 585-587, 2009.

[21] N. Salas, Development of a Delivery System and OpticalThermal Model for Laser Interstitial Thermotherapy of Breast Tumors, dissertation, University of Miami, Florida, FL, USA, 2007.

[22] M. Almadi, Optical Properties Measurements of Rat Muscle and Myocardium at 980 and $1860 \mathrm{Nm}$ Using Single Integrating Sphere Technique, Master thesis, University of Miami, Florida, FL, USA, 2014.

[23] G. Segelstein, The Complex Refractive Index of Water, Ph.D. dissertation, University of Missouri-Kansas City, Kansas, MO, USA, 1981.

[24] X. Ma, J. Q. Lu, R. S. Brock, K. M. Jacobs, P. Yang, and X.-H. Hu, "Determination of complex refractive index of polystyrene microspheres from 370 to $1610 \mathrm{~nm}$," Physics in Medicine and Biology, vol. 48, no. 24, pp. 4165-4172, 2003.

[25] I. D. Nikolov and C. D. Ivanov, "Optical plastic refractive measurements in the visible and the near-infrared regions," Applied Optics, vol. 39, no. 13, pp. 2067-2070, 2000.

[26] N. G. Sultanova, S. N. Kasarova, and I. D. Nikolov, "Characterization of optical properties of optical polymers," Optical and Quantum Electronics, vol. 45, no. 3, pp. 221-232, 2013. 

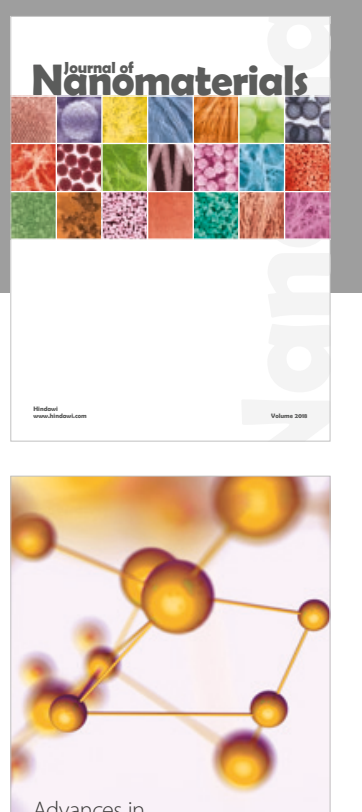

Physical Chemistry
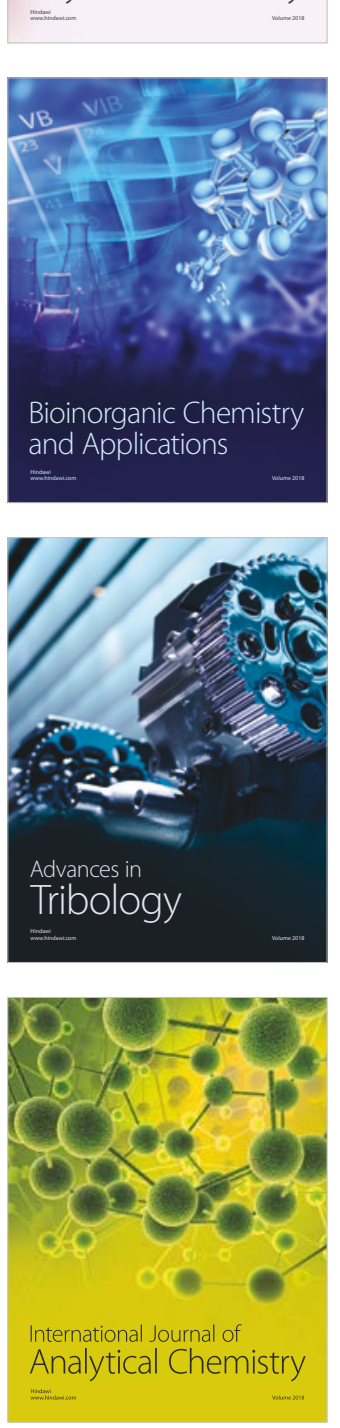

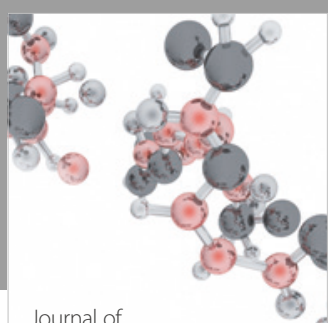

Analytical Methods

in Chemistry

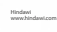

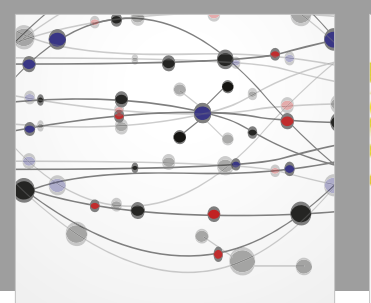

The Scientific World Journal

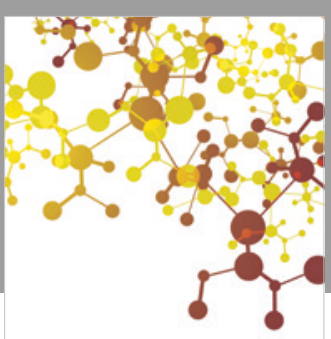

Journal of

Applied Chemistry
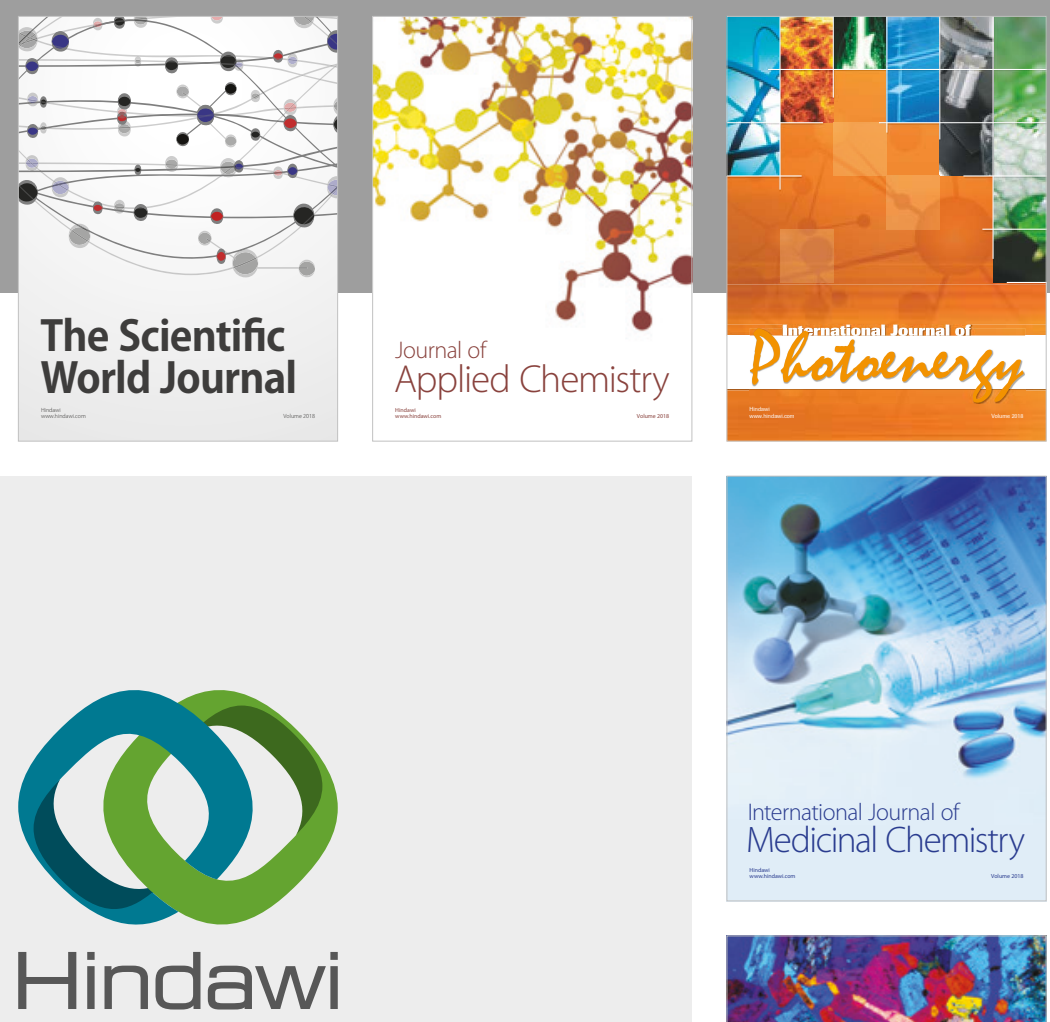

Submit your manuscripts at

www.hindawi.com
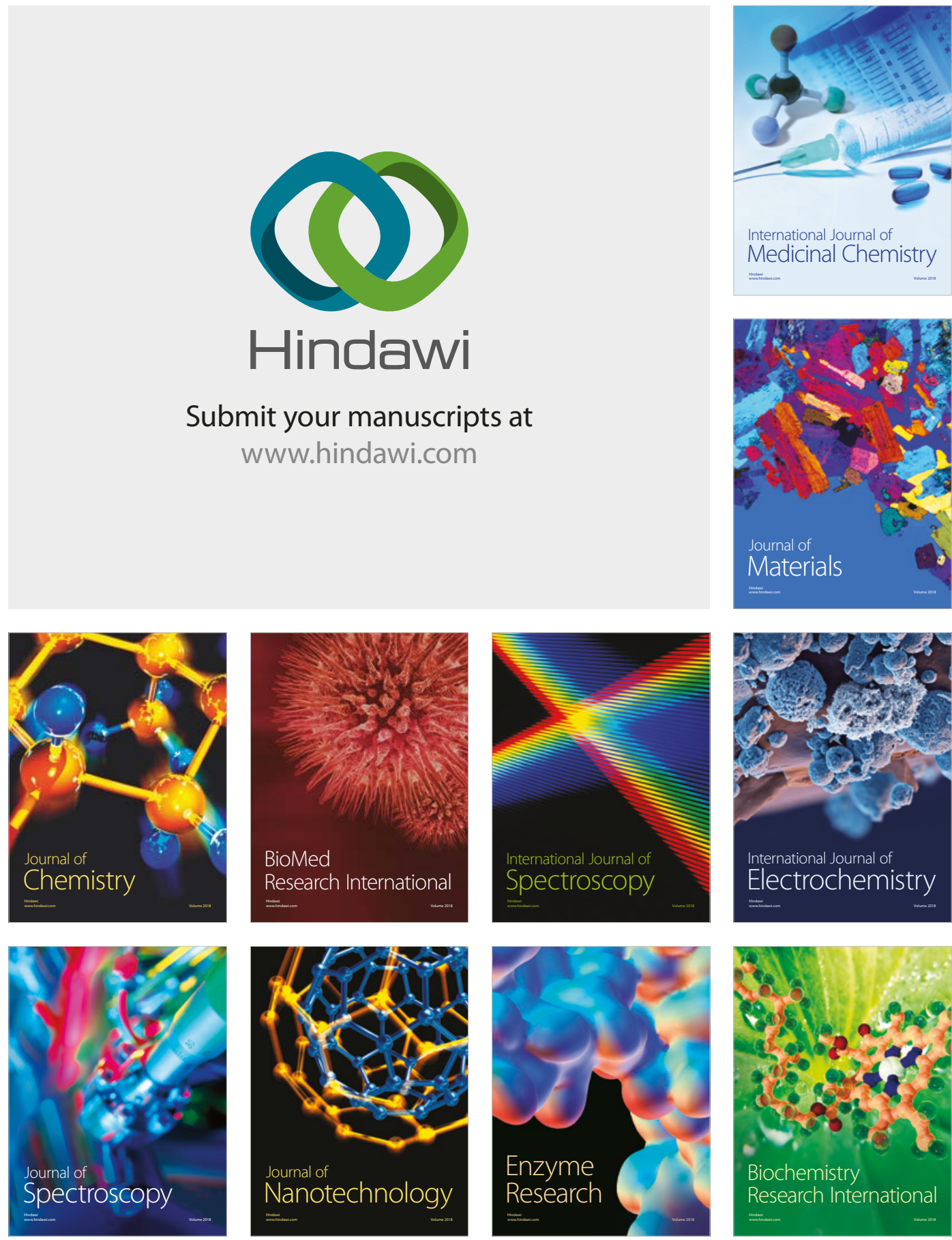
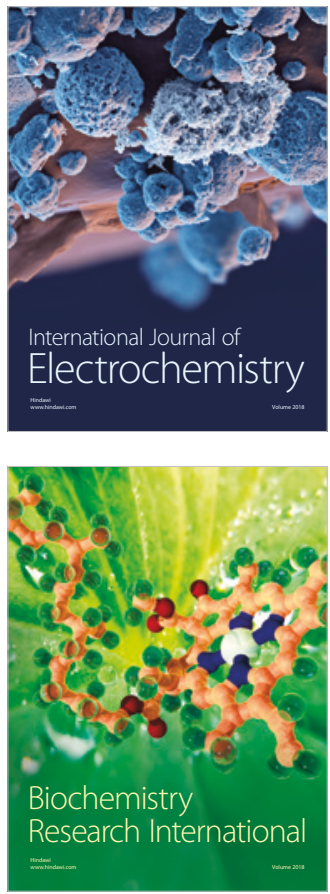